\title{
Image Processing for Face Recognition Rate Enhancement
}

\author{
Isra'a Abdul-Ameer Abdul-Jabbar \\ School of Computer and Information, Hefei University of Technology, Hefei 230009, \\ People's Republic of China \\ Computer Science Department, University of Technology, Baghdad, Iraq \\ Israa_ameer@yahoo.com
}

\begin{abstract}
In this paper, the impact of face image pre-processing in rising the face recognition rate is presented, considering the face images with low contrast, bad or dark lighting. Three preprocessing steps including image adjustment, histogram equalization and image conversion to Joint Photographic Experts Group (JPEG) or (JPG) and Bitmap (BMP) are used to enhance the contrast and the quality of face images respectively. For dimension reduction and feature extraction purposes many techniques are adopted such as Principle Component Analysis (PCA), Linear Discriminant Analysis (LDA), Kernel Principle Component Analysis KPCA and Kernel Fisher Analysis (KFA) and are used to evaluate the effect of illumination variations and image file formats on these techniques. Our results show that the proposed face databases in JPG and BMP formats produced good enhancement and increased the face recognition rate in all techniques when compared with AT\&T ORL face database.
\end{abstract}

Keywords: Adjust Image, Histogram equalization, Image conversion, Feature extraction, Face recognition rate

\section{Introduction}

Image pre-processing and normalization are important parts of face recognition techniques. Variations in lighting conditions produce dramatically decrease of recognition performance. If an image has low contrast and dark lighting places, its contrast and brightness should be improved. The common histogram equalization cannot correctly improve all parts of the image. When the face image has irregularly lighted conditions, some places on the resulted image will remain too dark or too bright. Face image has digital representation as any digital image; this mean has a binary representation of a two-dimensional image. The digital representation is an array of two dimensions called pixels. Each pixel has a numerical value which in monochromatic images appears as a grey level [1].

During the past two or three decades, many face recognition techniques have been proposed and reviewed in $[2,3,4,5]$. The main part of these techniques or systems is the feature extraction process. In this stage, an appropriate face representation is needed. This would be computationally functional and also powerful to possible intrinsic and extrinsic facial variations. Many feature extraction methods have been hard-done by face recognition systems including Principal Component Analysis (PCA) [2, 6], Linear Discriminant Analysis (LDA) [7], kernel methods [8], Eigenfaces [2], Fisherfaces [9], Laplacian faces [10], elastic bunch graph matching [11], neural networks[12] and support vector machine[13]. 
In face recognition literature, there are different face representation methods based on global features, including a big number of subspace-based methods and some spatialfrequency techniques [1]. In face recognition, individuals are identified by use of a large database of face images. In conventional appearance-based systems, the intensity of each pixel in a face image is entered as input feature. Because, there are many thousands of pixels in a face image [14], facial image data are always high-dimensional and significant computational time is required for the successful classification purpose. Thus the subspace methods, by projecting objects to a lower dimensional space, are commonly used. In practical cases, when the image dimension is very large, one is often forced to use linear techniques. Two important linear techniques for extracting characteristic features and also dimension reduction are PCA and LDA. Many researches focused on projective transforms. The essential part of these methods is creating feature vector for each face image, then classify the input face image in large database. Creating feature vector also has the utility of minimizing dimension of the input images [4]. Principal component analysis (PCA) method achieved the dimension reduction by projecting the original face image data onto lower dimensional subspace crossed by the best eigenvectors of the covariance matrix. Linear Discriminant Analysis (LDA) method looks for the projective axes on which the data points of two or more different classes are distant from each other, this mean LDA searches for the maximizing between class scatter, while constraining the data points of the same class to be as near to each other as possible, this mean searching for the minimizing within class scatter [7]. Kernel PCA and kernel fisher analysis are non linear form of PCA and LDA respectively. Several researchers proposed techniques based on spatial-frequency methods, such as Discrete Cosine Transform (DCT) and Fourier transform [12, 14, 15]. In these methods, face images are mapped to a lower frequency domain bands that have the most facial discriminating features and discarding high bands that having noise [14].

An important aspect in improving the performance of face recognition system is the enhancing of face image. The intended aim of face image enhancement is that the resulted images have better visual quality than the input one. Face image can be improved, by enhancing the brightness, contrast and resolution of image. This is a part of pre-processing stage that can affect the feature extraction and finally recognition performance. For instance in [16], the image enhancement has been considered in face recognition technique. Song et al. [17], calculates, prior to feature extraction stage, the illumination difference between right and left part of face. If there is a spacious amount of difference than take the mirror of average illuminated part.

The aim of this work is to study the effect of image pre-processing on increasing the face recognition rate and to study the impact of image file formats on these techniques. We will examine two formats represented by JPG and BMP file formats, also in this paper the effect of image enhancement on the face recognition are considered. For feature extraction stage, we used the PCA, LDA, KPCA and KFA. The emulation results show that our image enhancement noticeably increased the face recognition rate.

Section 2 explains the background; Section 3 describes the proposed enhancement approach. Sections 4 illustrate the experiments and results. Finally, the conclusions are discussed in section 5 .

\section{Background}

A common block diagram of face recognition and verification techniques is illustrated in the Figure 1. This diagram shows the training stage at top and the test stage at the top of the figure. The pre-processing steps include image resizing, histogram equalization and some other enhancement process. Then the scale vector constructed in the feature extraction stage is 
compared with the one of every face database class. If there is at least one face in database similar to the input test image, then the input face is recognized as "known", otherwise "unknown"[1].

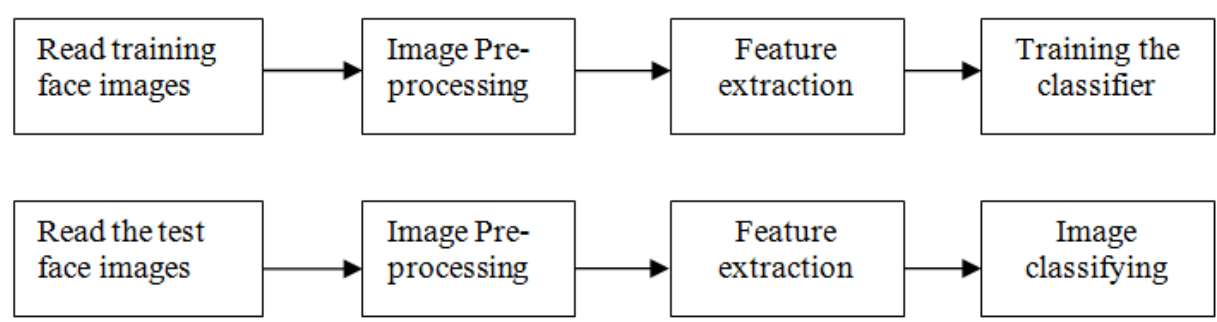

Figure 1. Block diagram of face recognition techniques

\subsection{Image Adjustment}

Image adjustment maps the intensity values in grayscale image I to new values in $\mathrm{J}$ such that $1 \%$ of data is saturated at low and high intensities of I. This increases the contrast of the output image J, Figure 2 shows the result of applying image adjust on the face image [14].
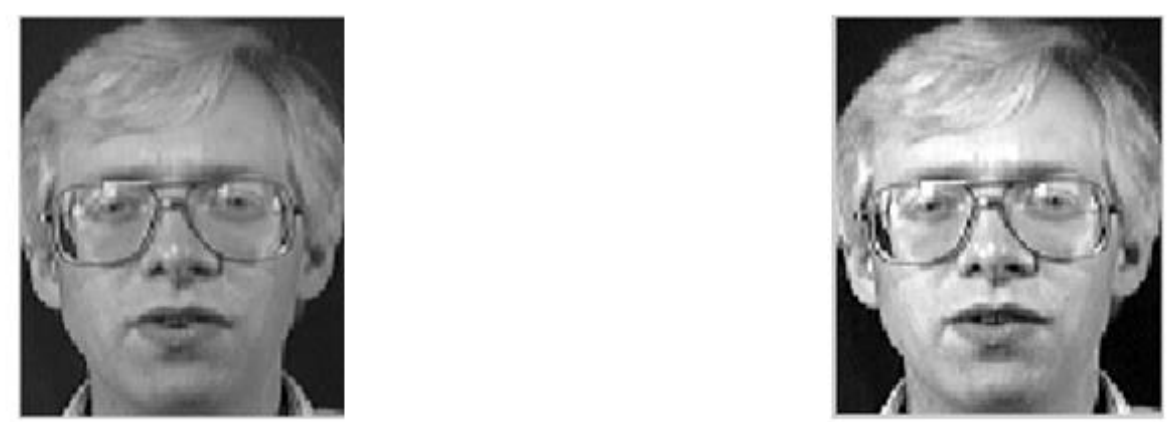

\section{Figure 2. Original face image is on the left, and the adjusted face image is on the right}

\subsection{Histogram Equalization}

The performance of face recognition techniques is also affected by illumination [16]. Histogram equalization is an effective method to deal with varying light conditions in large dataset of images. The problem is that the variation in illumination between images of distinct faces can be fewer than the variations between images of the same face under diverse illuminations conditions. It can even be displayed that illumination causes huge variation in face images than pose. At the very beginning of modern machine face recognition, it became very clear that different illumination in different images will be a problem [18]. Image preprocessing and normalization is played an important role in face recognition systems. Changes in lighting conditions will cause dramatic decreasing in the recognition performance [16].

Histogram equalization is a functional method in stretching the range of gray levels and expands the contrast of the image. It also makes the variation of the order of gray levels of the original image perfectly controllable. Therefore, it can improve image effectively [14, 16]. 
Figure 3 is the result of applying histogram equalization, it can be seen that the contrast of face image and background become more, and the look over of the face image is more evident.
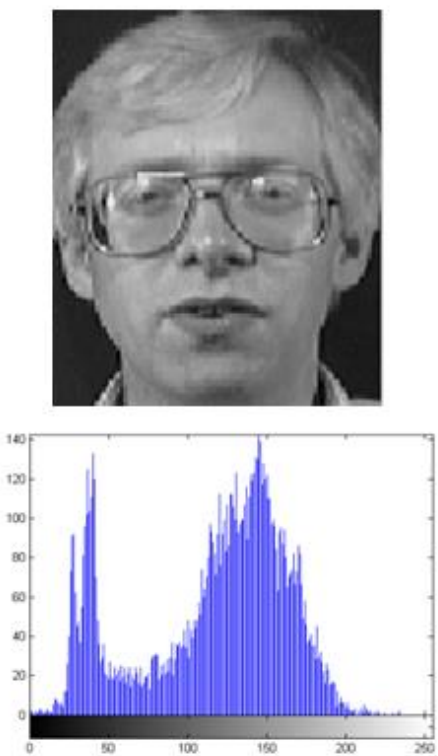
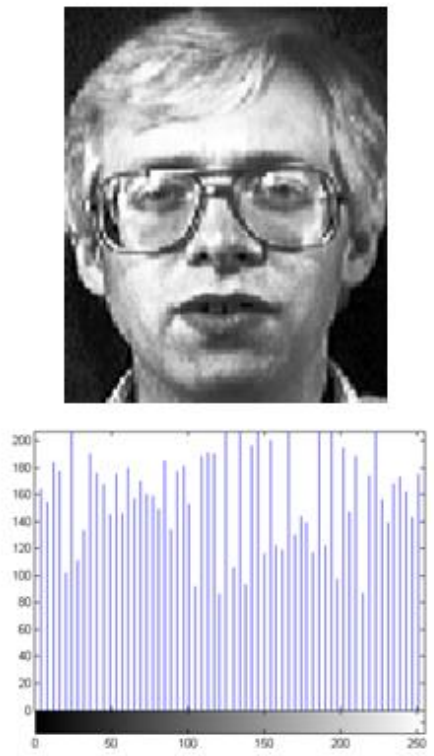

Figure 3. Original face image is on the left, and the histogram equalization face image is on the right

\subsection{Image File Formats}

The most common face image file formats, the most important for face recognition systems. Today many face databases are in different formats PGM, BMP, JPG, GIF and many other formats, these are not the only choices of course, but they are good and reasonable choices for security purposes [19], for this reason we will also give a brief description to the image file formats that used in our paper.

PGM (Portable Gray Map) is a standard bitmap format consisting of a four lines header, the data stored as unsigned char type; consist of 8-bit per pixel with maximum 256 gray scale levels. The structure of a PGM images file consists of four lines header: the first line containing the directory of the image file and identifying the file as PGM. The second line is the comment line. The third line gives information related to the number of rows and columns of the data in the file, and the fourth line identifies the maximum gray level contained in the image. The data of PGM image follows the header information and is written in text or binary format as pixel values, this mean the data is in raster order. PGM file images are generally used for displaying and printing purposes and can be opened on many packages that are commercially available like UNIX.

BMP (Bit Map Picture) is a raster graphics image; its rank is suitable for Microsoft Windows Bitmap. This format converts an image pixel bit by bit. It is the only graphics format used where compression actually enlarges the file.

JPEG (Joint Photographic Experts Group) uses lossy compression, but its rank is suitable for higher quality and larger files, or lower quality and smaller files. JPG is used for photo images, and it is not good choice for most graphics or text data. It can be recognized by all 
Web browsers and it is the best choice today. It is providing only these two formats RGB- 24bits (8-bit for each color) and Grayscale - 8-bits.

\section{The Proposed Enhancement Approach}

In this section, we will describe the proposed enhancement approach, we used ORL database of PGM $(92 \times 112)$ file format. In our work, two specific image processing functions are applied on this database which are suitable for contrast enhancement including adjust face image and the applying of histogram equalization, then the image is transformed to JPG and BMP file formats to create two databases entered to feature extraction process by using several face recognition techniques represented by PCA, LDA, KPCA and KFA, this is to evaluate the performance of these techniques after the enhancement process.

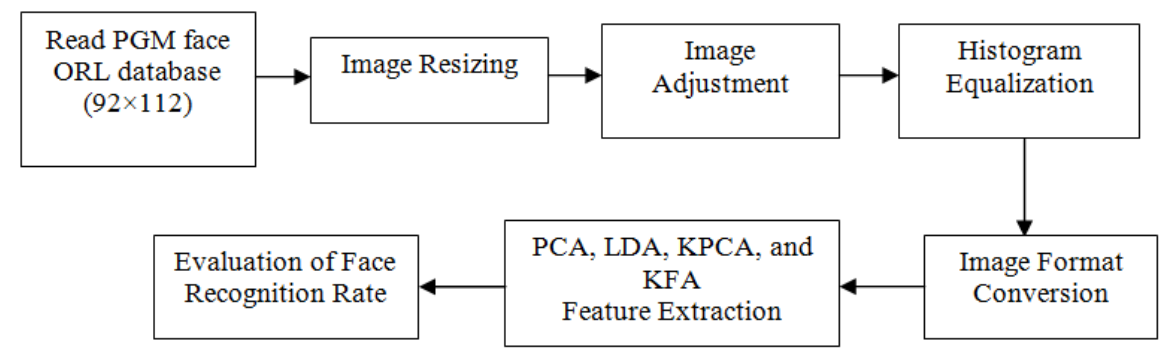

Figure 4. The proposed face image enhancement and recognition block diagram

\subsection{Image Adjustment}

The first pre-processing enhancement step in our work is the image adjustment step, Figure 5 shows an example of implemented the image adjustment on three samples of face images in ORL face database of PGM format, in the first row of Figure 5 is the original input face image and in the second row is the adjusted one.

\subsection{Histogram Equalization}

Histogram equalization generally is a common method for enhancing the low contrast and the appearance of a face image by effectively increasing the distance among the most frequently appeared intensity values through a nonlinear mapping function and is usually histogram modification approach [15]. The intended aim of histogram equalization is to allow pixels in the areas with lower contrast to seem in a higher contrast [14].

In our work we applied histogram equalization after the image adjustment step and the third row of Figure 5 shown the result of entering the adjusted image to histogram equalization to get (the adjust-histogram) image. 

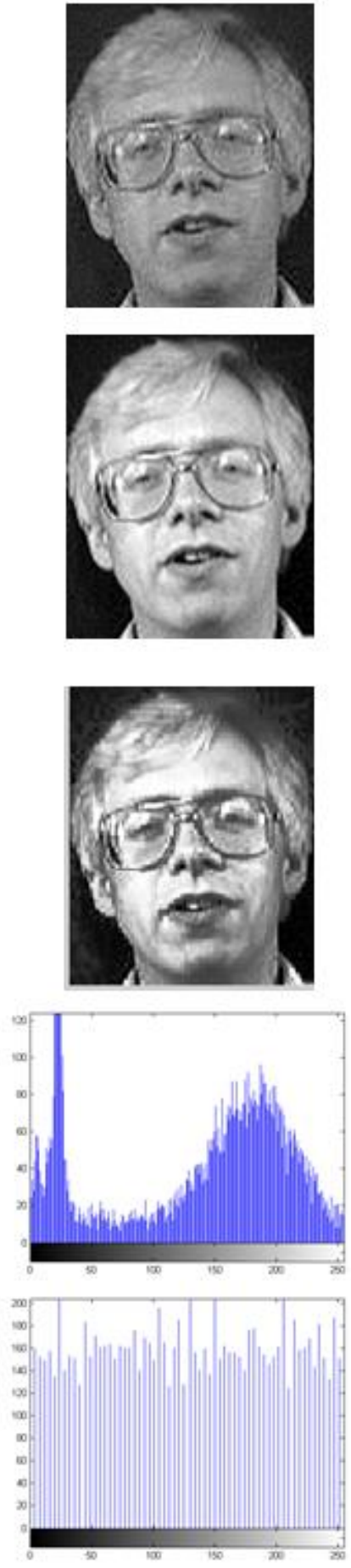
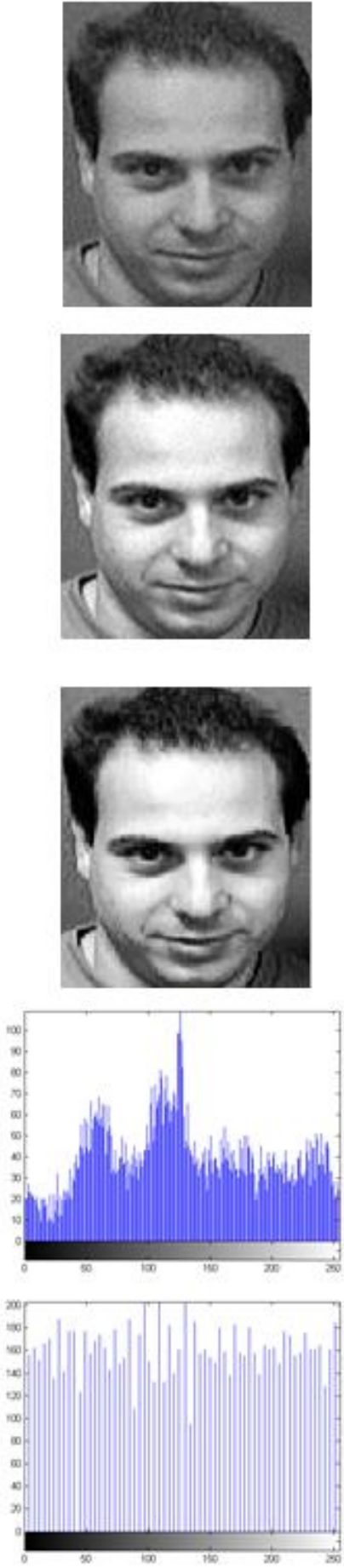
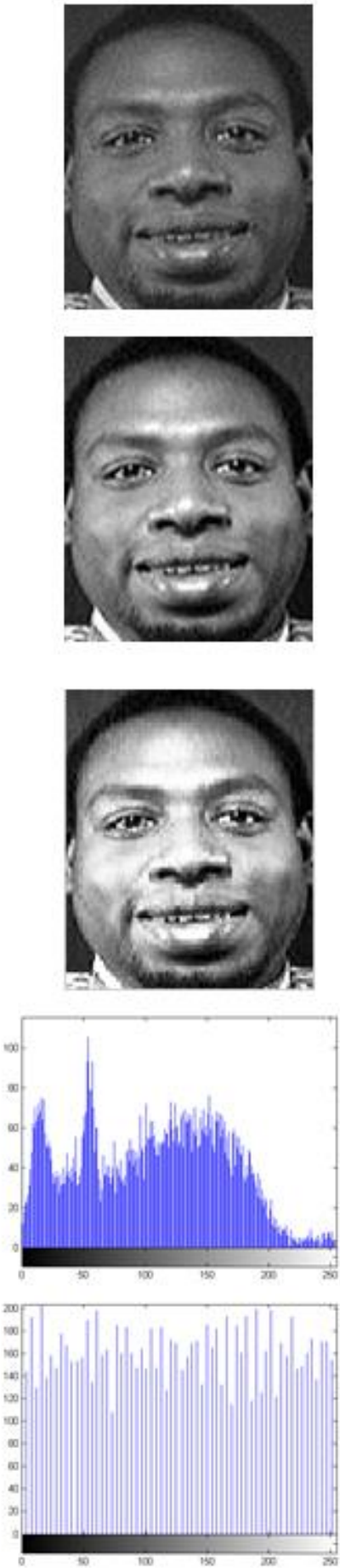

Figure 5. Series of face processing steps, in the first row is the original image, in the second row is the adjusted image, the third row and below is the result of adjust-histogram image 


\subsection{Image Conversion}

The third stage that follows the image adjustment and histogram equalization is the conversion of PGM face image file format to JPG and BMP formats to generate two face image databases. Figures 6 and Figure 7 show the conversion of the resulted adjustedhistogram image to JPG and BMP respectively, they are looked similar, but really they have different format.

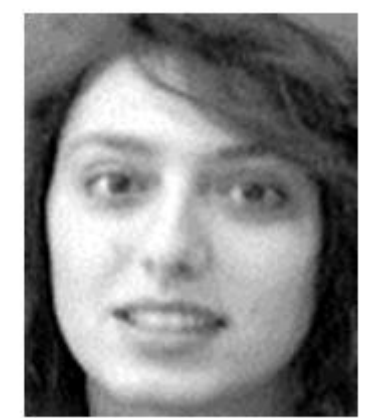

Figure 6. JPG adjust-histogram image

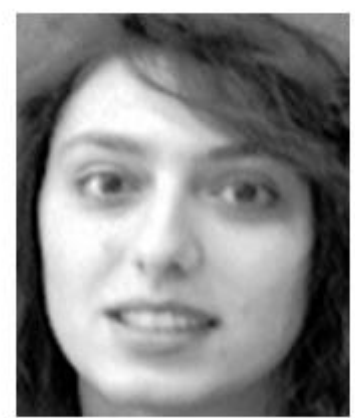

Figure 7. BMP adjust-histogram image

\subsection{Face Recognition Feature Extraction Techniques}

All databases (the original and the proposed ones) are examined under a statistical feature extraction process by using many face recognition techniques PCA, LDA, KPCA and FA, and passed across many steps including: creating data matrix, creating training images space, calculating mean face from training images, normalization and images dimensionality reduction. The Pretty helpful Development $(\mathrm{PhD})$ face recognition toolbox by Struc [20, 21] is used to evaluate our databases, since it contains some of the most popular face recognition techniques that used for face recognition purposes. The general steps of face recognition techniques can be summarized as follows:

Step 1: Load images from a database. In our case, the original PGM ORL database and the proposed databases of BMP and JPG are used.

Step 2: Partition data into training and test sets. In our case, the first 3 images of each subject will serve as the training/gallery/target set and the remaining 7 images will serve as test/evaluation/query images.

Step 3: Compute training and test feature vectors using the technique in choice. In our case we use different algorithms for feature extraction (PCA, LDA, KPCA, and FA) and therefore, first compute the subspace using the training data from the ORL database.

Step 4: Compute matching scores between gallery/training/target feature vectors and test/query feature vectors. In our case we use the cosine similarity for computing similarity matrix.

Step 5: Evaluate the results under criteria of face recognition rate.

\section{Experiments and Results}

In our work we evaluate our enhancement approach on the performance of PCA, LDA, and KFA techniques after the conversion of the enhanced ORL face database of 400 PGM file format to JPG file format. 
Table 1 shows the comparison result between the original ORL database of PGM file format with our proposed enhanced JPG database, three methods are used for this comparison including PCA, LDA and KFA when 400 face images are used.

Table 2 shows the result of comparison between same databases that used in Table 1 but on sample of 200 face image are used, and the recognition rates are evaluated with five techniques including PCA with two distance metrics (Euclidean and Mahcos distances), LDA, KPCA and KFA.

Table 1. Comparison between PGM ORL database and our enhanced JPG database of 400 face images implemented with PCA, LDA, KFA

\begin{tabular}{|c|c|c|c|}
\hline Database Used & PCA +Mahcos & LDA & KFA \\
\hline ORL PGM db [20,21 ] & $66.07 \%$ & $86.07 \%$ & $85.07 \%$ \\
\hline Adjust-hist. JPG db & $75.00 \%$ & $91.43 \%$ & $86.43 \%$ \\
\hline
\end{tabular}

\section{Table 2. Comparison between PGM ORL database and our enhanced JPG database with image adjustment and histogram equalization when implemented on 200 face images}

\begin{tabular}{|c|c|c|c|c|c|}
\hline Database Used & Std. PCA & PCA +Mahcos & LDA & KPCA & KFA \\
\hline ORL PGM db & $77 \%$ & $79.29 \%$ & $93.57 \%$ & $38.57 \%$ & $91.43 \%$ \\
\hline Adjust - hist. JPG db & $82 \%$ & $90.00 \%$ & $97.75 \%$ & $70.00 \%$ & $92.14 \%$ \\
\hline
\end{tabular}

Also, we converted all face images of ORL database to BMP format after the implementation of enhancement processing (image adjustment and histogram equalization); we evaluated the performance of the enhanced BMP database and compared it with the original ORL database.

Table 3 shows the comparison result between original ORL database of PGM file format with our proposed BMP database, three methods are used for this comparison including PCA, LDA and KFA when applied on 400 face images.

Table 4 shows the result of comparison between same databases that used in Table 3 but on sample of 200 face image implemented and the recognition rates are evaluated with five feature extraction tecniques including PCA with two distance metrics (Euclidean and Mahcos distances), LDA, KPCA and KFA.

Table 3. comparison between PGM ORL database and our enhanced BMP database of $\mathbf{4 0 0}$ face images implemented with PCA, LDA, KFA

\begin{tabular}{|c|c|c|c|}
\hline Database Used & PCA +Mahcos & LDA & KFA \\
\hline ORL PGM db [20][21 ] & $66.07 \%$ & $86.07 \%$ & $85.07 \%$ \\
\hline Adjust-hist. BMP db & $74.64 \%$ & $91.43 \%$ & $86.43 \%$ \\
\hline
\end{tabular}

Table 4. comparison between PGM ORL databse and our enhanced BMP database with image adjustment and histogram equalization when implemented on 200 face images

\begin{tabular}{|c|c|c|c|c|c|}
\hline Database Used & Std. PCA & PCA +Mahcos & LDA & KPCA & KFA \\
\hline ORL PGM db & $77 \%$ & $79.29 \%$ & $93.57 \%$ & $38.57 \%$ & $91.43 \%$ \\
\hline Adjust - hist. BMP db & $78 \%$ & $90.00 \%$ & $96.34 \%$ & $69.29 \%$ & $92.14 \%$ \\
\hline
\end{tabular}




\section{Conclusions}

The intended aim of face recognition systems is to retrieve face images which have high similarity to a specific query face image in large database. The extracted face images can be used for many applications, such as visual surveillance, criminal face verification, retrieving specific faces from the web, and photo management.

In this paper, image enhancement process to improve the face recognition rate is presented. The novelty of this paper focuses on the enhancement of face recognition based on face image enhancement, image file format, the combine of PCA, LDA, KPCA and KFA feature extraction and dimension reduction. At the first step, the faces images are adjusted with image adjustment, then, the images is processed with histogram equalization, then the image converted from PGM to JPG and BMP to get two face databases with high quality, each of them with 400 face image. The PCA is used to reduce the dimension of face images with two distance measures Euclidean distance and Mahcos distance, and the other three techniques LDA, KPCA, and KFA are used with Mahcos distance, the results obtained using this approach indicate sharper edges, clearer metrics and give more details that led to the superior performance than the original PGM AT\&T ORL database.

The main advantage of our enhancement approach is the ease of implementation, we found JPG and BMP databases have the same recognition rate with PCA and KFA, while JPG database has recognition slightly higher by $(1.5 \%, 0.71 \%)$ than BMP database with LDA, KPCA. In other word the two databases achieved higher performance than the original ORL database with four feature extraction techniques and with two distance metrics, and because JPG and BMP file formats are better than PGM face image format, both formats can be trusty used for face recognition systems in airports, monitoring systems, verifying of criminals in the police office and many different security measures.

The other file formats such as TIFF, PNG and GIF image file formats produced the same performance when used as face databases and implemented on the same recognition techniques, also we found all these formats have recognition rate equal to that of BMP database, this is because the lossless formats of these images and this is the main reason that we didn't mention these formats in our paper.

\section{Acknowledgements}

The work is supported by the NSFC-Guangdong Joint Foundation (Key Project) under Grant No. U1135003 and the National Natural Science Foundation of China under Grant No. 61070227.

\section{References}

[1] N. Amani, A. Shahbahrami and M. Nahvi, "A New Approach for Face Image Enhancement and Recognition", International Journal of Advanced Science and Technology, vol. 52, (2013).

[2] M. Turk and A. Pentland, "Eigenfaces for recognition. Journal of Cognitive Neuroscience", vol. 3, no. 1, (1991).

[3] A. J. Colmenarez and T. S. Huang, "Face Detection and Recognition. Berlin: Springer-Verlag", Series F: Computer and Systems Sciences, vol. 163, (1998), pp. 174-185.

[4] C. Gurel, "Development of a Face Recognition System”, Master of Science thesis, Atilim University, (2011).

[5] G. Wang and Z. Ou, "Face Recognition Based on Image Enhancement and Gabor Features", Proceedings 6th World Congress on Intelligent Control and Automation, Dalian, China, (2006) June, pp. 9761-9764.

[6] K. Kim, Editor, "Face recognition using principle component analysis", International Conference on Computer Vision and Pattern Recognition, USA, (1996).

[7] H. Yu and J. Yang, "A direct LDA algorithm for high dimensional data-with application to face recognition", The Journal of Pattern Recognition, vol. 34, (2001), pp. 2067-2070. 
[8] P. Penev and J. Atick, "Local Feature Analysis: A General Statistical Theory for Object Representation", Network Computation in Neural Systems, vol. 7, (1996).

[9] P. N. Belhumeur, J. P. Hespanha and D. J. Kriegman, "Eigenfaces versus fisherfaces: Recognition using class specific linear projection”, IEEE Trans. Pattern Anal. Mach. Intell., vol. 19, no. 7, (1997).

[10] X. He, S. Yan, Y. Hu, P. Niyogi and H. -J. Zhang, "Face recognition using Laplacianfaces. IEEE Trans. on PAMI, vol. 27, no. 3, (2005).

[11] L. Wiskott, J. Fellous, N. Kruger and C. Malsburg, "Face Recognition by Elastic Bunch Graph Matching", IEEE Tran. on Pattern Analysis and Machine Intelligence, vol. 19, no. 7, (1997).

[12] S. Mallat, "A wavelet tour of signal processing”, 3rd edition, Elsevier (Singapore) Pte Ltd, (2009).

[13] J. Huang, X. Shao and H, "Wechsler", Face Pose Discrimination Using Support Vector Machines (SVM). Proceeding of 14 th conference on pattern Recognition, (ICPR 98), Brisbane, Qld, (1998) August.

[14] R. C. Gonzalez and R. E. Woods, "Digital Image Processing $3^{\text {rd }}$ edition", Prentice Hall, Upper Saddle River, NJ, (2008).

[15] A. K. Jain, "Fundamentals of Digital Image Processing”, Prentice Hall, New Jersey, (1989).

[16] V. V. Starovoitov, D. I. Samal and D. V. Briliuk, "Image Enhancement for Face Recognition", Submitted to International Conference on Iconics, St.Petersburg, Russia, (2003).

[17] Y. Song, Y. Kim, U. Chang and H. B. Kwon, "Face recognition robust to left/right shadows; facial symmetry", Pattern Recognition, vol. 39, (2006).

[18] K. Delac, M. Grgic and T. Kos, "Sub-Image Homomorphic Filtering Technique for Improving Facial Identification under Difficult Illumination Conditions", International Conference on Systems, Signals and Image Processing, Budapest, Hungary, (2006).

[19]W. Fulton. Image File Formats - JPG, TIF, PNG, GIF, Which to use? Web page available at http://www.scantips.com/basics09.html, (2013).

[20] Struc and N. Pave`si'c, "The complete gabor-fisher classifier for robust face recognition", EURASIP Advances in Signal Processing, doi =10.1155 / 2010 / 847680, (2010), p. 26.

[21] Struc and N. Pave si'c, "Gabor-based kernel partial-least-squares discrimination features for face recognition”, Informatica (Vilnius), vol. 20, no. 1, (2009), pp. 115-138.

\section{Author}

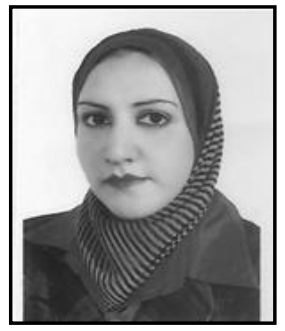

\section{Isra'a Abdul-Ameer Abdul-Jabbar}

Received her B.S. degree in 2003 and M.Sc. degree in 2006 in Computer Science both were from Department of Computer Science, University of Technology, Baghdad, Iraq. She is now a $\mathrm{PhD}$. candidate at the School of Computer and Information in Hefei University of Technology, Hefei city, China. Her research interests include Face Recognition Systems and Image Processing. 